\title{
Classical Loschmidt echo in chaotic many-body systems
}

\author{
Gregor Veble ${ }^{1,2}$ and Tomaž Prosen ${ }^{1}$ \\ ${ }^{1}$ Physics Department, FMF, University of Ljubljana, Ljubljana, Slovenia \\ 2 Center for Applied Mathematics and Theoretical Physics, University of Maribor, Maribor, Slovenia
}

(Dated: November 17, 2018)

\begin{abstract}
General theoretic approach to classical Loschmidt echoes in chaotic systems with many degrees of freedom is developed. For perturbations which affect essentially all degrees of freedom we find a doubly exponential decay with the rate determined by the largest Lyapunov exponent. The scaling of the decay rate on the perturbation strength depends on whether the initial phase-space density is continuous or not.
\end{abstract}

PACS numbers: 05.45.Jn

Understanding macroscopic irreversibility from reversible miscroscopic evolution laws is one of the fundametal controversies of statistical mechanics, put forward by Loschmidt [1]. The problem has been reformulated in terms of the so called Loschmidt echo or fidelity $[2,3,44,5,6]$, mainly in the context of quantum chaos and quantum information, and only more recently $[6,7,8]$ in the context of classical dynamics. In [9] it was shown that dynamics in the echo picture, which is a composition of perturbed forward and unperturbed backward Hamiltonian evolution in time, can again be written as forward Hamiltonian evolution. The Hamiltonian in this case becomes time dependent and is merely the perturbation part of the original Hamiltonian evaluated at a point obtained by the forward unperturbed evolution. Analyzing the motion in the echo picture for the case of Anosov systems showed that, for sufficiently long times, it can be separated into a composition of independent exponentially accelerated one-dimensional motions, each such motion locally corresponding to a Lyapunov vector direction of the original, unperturbed system. Motion along each such (unstable) direction could be well described statistically, using a kernel whose shape is time independent but whose width increases as $\sim e^{\lambda_{i} t}$ where $\lambda_{i}$ is the corresponding Lyapunov exponent. The theory [9] shows that such an evolution leads to the Lyapunov decay of fidelity for the case of a system with one degree of freedom, confirming results of Refs. [6, 7, [8]. For systems with more than a single degree of freedom a cascade of exponential decays with increasing decay rates was predicted and observed.

Here we extend the results to systems with many degrees of freedom. While a similar theroy may still be applied, the results are significantly different as the regime which is considered perturbative in systems with few degrees of freedom is actually the dominant one for systems with a large number of freedoms. What we find is that the decay of fidelity for systems with a large number of degrees of freedom decays as a doubly exponential function of time for the case of a global perturbation (afftecting all degrees of freedom), and the scaling of decay rate on the strength of perturbation depends on whether the initial phase space density is continuous or not. In another case of a local perturbation we find and justify a system dependent form of decay that falls between the exponential and doubly exponential behaviors.

In a system with $N$ phase space variables fidelity is defined as the overlap

$$
f(t)=\int \mathrm{d}^{N} \boldsymbol{x} \rho_{0}(\boldsymbol{x}) \rho_{t}(\boldsymbol{x}),
$$

where $\rho_{0}$ is the initial phase space density, $\boldsymbol{x}$ is a phase space point and $\rho_{t}$ is the echo density as obtained by a composition of a perturbed forward evolution for time $t$ with Hamiltonian $H=H_{0}+\delta V, \delta$ is the strength of perturbation, and an unperturbed backward evolution, again for time $t$, with Hamiltonian $H_{0}$. As shown in [9], under the assumption that the time is long enough in order for the echo dynamics to start to behave statistically, the echo density can be written as

$$
\rho_{t}(\boldsymbol{y})=\int \mathrm{d}^{N} \boldsymbol{y}^{\prime} \rho_{0}\left(\boldsymbol{y}+\boldsymbol{y}^{\prime}\right) \prod_{i=1}^{N} \frac{1}{\sigma_{i}} K_{i}\left(\frac{y_{i}^{\prime}}{\sigma_{i}}\right),
$$

where new variables $y_{i}$ are introduced (see [9]) that give the coordinates along the stable and unstable local Lyapunov vectors. The kernels $K_{i}$ are time independent with their typical widths of the order of 1 , the time dependence being present in the widths $\sigma_{i}=\delta \gamma_{i} \exp \left(\lambda_{i} t\right)$ for the unstable and $\sigma_{i}=\delta \gamma_{i}$ for the stable directions, where $\delta$ is the strength of the perturbation and $\lambda_{i}$ the corresponding Lyapunov exponent. The factor $\gamma_{i}$ in the expression of $\sigma_{i}$ is determined by the typical values of the phase space variable $W_{i}(\boldsymbol{x})=\boldsymbol{e}_{i}(\boldsymbol{x}) \cdot \nabla V(\boldsymbol{x})$, where $\boldsymbol{e}_{i}$ is the corresponding Lyapunov vector defined as in [9]. Fidelity can be rewritten in the new coordinates as $f(t)=\int \mathrm{d}^{N} \boldsymbol{y} \rho_{0}(\boldsymbol{y}) \rho_{t}(\boldsymbol{y})$.

Let us assume now that the initial density is a product $\rho_{0}(\boldsymbol{y})=\prod_{i=1}^{N} \rho_{0}^{(i)}\left(y_{i}\right)$, for example being a characteristic funciton on a hypercube, or a multidimensional Gaussian, in coordinates $y_{i}$. In this case the subsequent analysis is very much simplified and we see no reason for the results to be different in the general cases, as indeed the numerics, which does not correspond to initial product denisty, shows. Under this assumption fidelity itself can be written as a product of contributions from different individual Lyapunov directions, $f(t)=\prod_{i=1}^{N} f_{i}(t)$. Each such contribution is of 
the form $f_{i}(t)=\int \mathrm{d} y \rho_{0}^{(i)}(y) \rho_{t}^{(i)}(y)$, where $\rho_{t}^{(i)}(y)=$ $\frac{1}{\sigma_{i}} \int \mathrm{d} y^{\prime} \rho_{0}^{(i)}\left(y+y^{\prime}\right) K_{i}\left(\frac{y^{\prime}}{\sigma_{i}}\right)$. The initial density contributions $\rho_{0}$ can be considered to be square normalized, and we may write

$f_{i}(t)=1-\frac{1}{2 \sigma_{i}} \int \mathrm{d} y \mathrm{~d} y^{\prime}\left[\rho_{0}^{(i)}(y)-\rho_{0}^{(i)}\left(y+y^{\prime}\right)\right]^{2} K_{i}\left(\frac{y^{\prime}}{\sigma_{i}}\right)$.

Introducing $D_{i}\left(y^{\prime}\right)=\int \mathrm{d} y\left[\rho_{0}^{(i)}(y)-\rho_{0}^{(i)}\left(y+y^{\prime}\right)\right]^{2}$ we may see that the behavior of $f(t)$ will be markedly different depending on whether the function $\rho_{0}$ is continuous or not. In the continuous case the function $D_{i}\left(y^{\prime}\right) \propto y^{\prime 2}$ for small $y^{\prime}$, whereas in the discontinuous but piecewise continuous case the behavior is $D_{i}\left(y^{\prime}\right)=G_{i}\left|y^{\prime}\right|$, where $G$ represents the sum of all squares of the discontinuity gaps of $\rho_{0}^{(i)}$. From this it follows that to the lowest order of $\sigma_{i}=\delta e^{\lambda_{i} t}$,

$$
1-f_{i}(t) \approx c_{i} \sigma_{i}^{\beta}=c_{i}\left(\delta \gamma_{i}\right)^{\beta} \exp \left(\beta \lambda_{i} t\right)
$$

where $\beta=1$ for the discontinuous case, and $\beta=2$ for the continuous case, and $c_{i}$ is a constant.

In the case of many degrees of freedom fidelity is a product of contributions of many separate unstable directions. Even if individual contributions $f_{i}(t)$ do not deviate significantly from 1 that may not be the case for the product of all $f_{i}$. When $N$ becomes very large we may therefore expect the regime where equation (11) is valid to describe the behavior of fidelity for all times until it becomes insignificantly small, at least in the cases where a significant fraction of unstable directions contribute to the decay of fidelity. The resulting expression is thus

$$
f(t)=\prod_{i=1}^{N}\left(1-c_{i}\left(\delta \gamma_{i}\right)^{\beta} \exp \left(\beta \lambda_{i} t\right)\right)
$$

where the product is taken along all the unstable directions $i, c_{i}$ are positive coefficients depending on the shape of the initial density $\rho_{0}$ and the details of the perturbation $V$ and $\beta=1,2$, depending whether the initial density is discontinuous, or continuous, respectively.

The behavior of fidelity depends on a few considerations. Here we limit ourselves to the cases of initial phase space densities that are not constant (inpedependent) with respect to any of the phase space variables. Another important distinction is the nature of the perturbation. The perturbation can be either local in nature, meaning that it significantly affects only a finite number of degrees of freedom of the system, or they can be extended such that each degree of freedom is affected equally on average.

In the case of an extended perturbation, each component of the gradient of the perturbation potential $\nabla V$ is of the order of 1, i.e. independent of $N$. As it may be argued that the norm of the Lyapunov vector $\boldsymbol{e}_{i}$ is independent of $N$, the magnitude of the variable $W_{i}$ can be estimated as $\left(\boldsymbol{e}_{i} \cdot \nabla V\right)^{2}=\left(\sum_{j=1}^{N}\left[\boldsymbol{e}_{i}\right]_{j} \partial_{j} V\right)^{2} \approx$

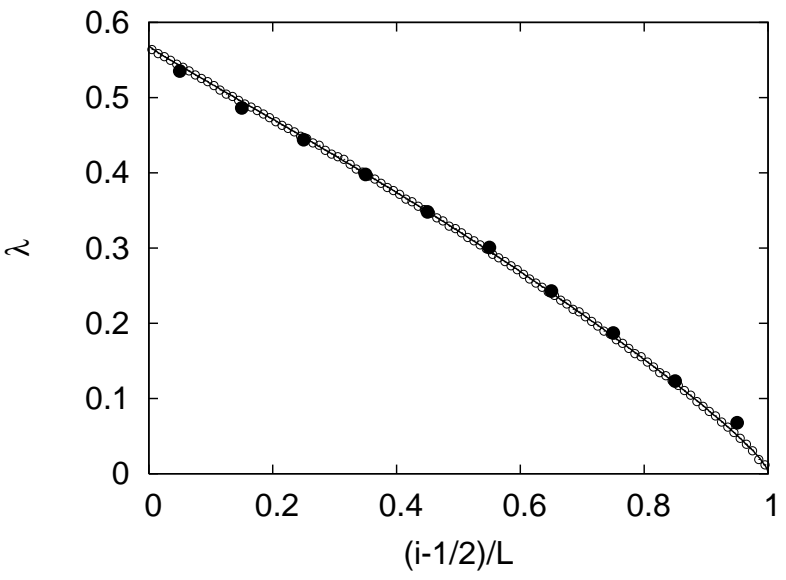

FIG. 1: The Lyapunov spectrum of the kicked Ising chain as calculated after $10^{4}$ kicks for $L=10$ (full circles), 100 (empty circles) and 1000 (line) spins sorted from the largest to lowest positive value (the negative and 0 values of the spectrum are omitted). On the horizontal axis we give $(i-1 / 2) / L$, where $i$ is the consecutive index of the Lyapunov exponent.

$\sum_{j=1}^{N}\left[\boldsymbol{e}_{i}\right]_{j}^{2}\left(\partial_{j} V\right)^{2}=\mathcal{O}(1)$. The above estimate is done by assuming that the components of the Lyapunov field vector are not correlated. This estimate gives the magnitude of the factor $\gamma_{i}$ in equation (2). In that equation, the most relevant contributions to the decay are those with the highest Lyapunov exponents. As numerical evidence below suggests, for uniform systems the Lyapunov spectrum in the thermodynamics limit $N \rightarrow \infty$ has a limiting distribution of Lyapunov exponents, with an existing maximum exponent $\lambda_{\max }$. From this it follows that in an interval $\left[\lambda_{\max }-\Delta \lambda, \lambda_{\max }\right]$ there will exist $\propto \Delta \lambda N$ Lyapunov exponents in this interval. The number $n$ of relevant factors that contribute to the decay of fidelity as given in (2) therefore scales linearly with $N$. Assuming that the factors $c_{i}\left(\delta \gamma_{i}\right)^{\beta}$ in equation (2) have a well defined average and are statistically independent, when $N$ becomes very large equation (2) can be written as

$$
f(t)=\exp \left(-\alpha N \delta^{\beta} \exp \left(\beta \lambda_{\max } t\right)\right),
$$

where $\beta=1$ or 2 . The decay of fidelity in many body systems with global perturbations is therefore expected to decay as a doubly exponential function, with the decay being stronger with increasing $N$. This is the main result of this Letter.

When considering the case of a local perturbation more care needs to be taken. Because the perturbation is local, the variables $W_{i}$ that determine the spreading of densities along individual Lyapunov directions are highly correlated amongst themselves. Namely, the Lyapunov vectors corresponding to the exponents close to the maximum one have a very similar structure on the local scale (in the sense of neighbouring degrees of freedom) and therefore the corresponding different phase space variables $W_{i}$ are, according to their definition, all very similar. This means that, for very short times, the contri- 


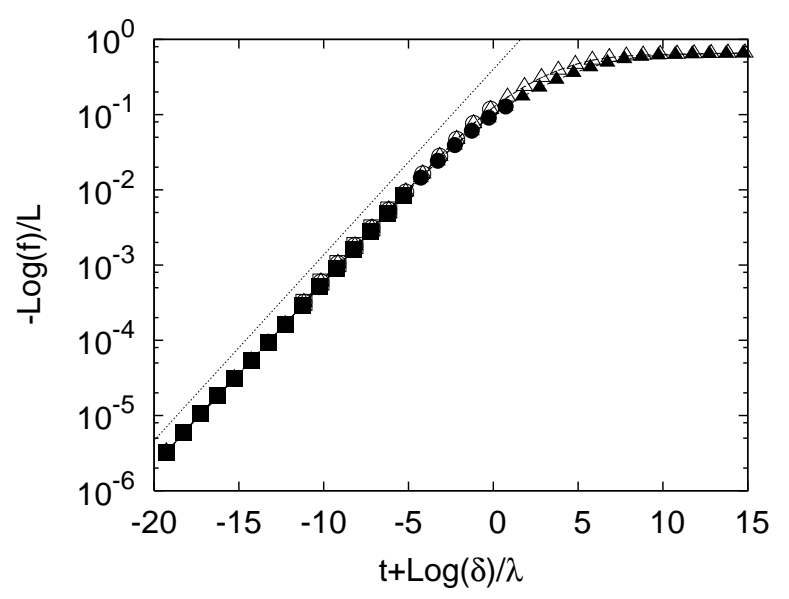

FIG. 2: Decay of fidelity as a function of time for the case of the discontinuous distribution. We divide the logarithm of fidelity by $L$, show it in log-scale, and shift the time by $\log (\delta) / \lambda_{\max }$. We show the data for $L=10$ (triangles), 100 (circles) and 1000 (squares) with perturbation strengths $\delta=10^{-3}$ (empty symbols) and $10^{-5}$ (full symbols). The number of trajectories was $n_{t}=10^{7}$ for the cases of $L=10,100$ and $n_{t}=10^{6}$ for $L=1000$. We may see that in the regime of validity of equation (3), as shown by the dotted line for $\beta=1$ with an arbitrarily chosen constant $\alpha$, all data scale to the same curve in the diagram. The deviations near the saturation values are due to different times of dynamics of the system and therefore a different decay of correlations (see [8]), this regime however moves to the values of fidelity approaching 0 with $L \rightarrow \infty$.

butions to the spreading of density along different Lyapunov directions cannot be considered to be independent of each other, but rather the spreading is effectively onedimensional along the average of the Lyapunov directions in phase space. This average direction corresponds to the (local) degree of freedom in which the perturbation is being applied. At longer times, due to the coupling between the neighbouring degrees of freedom, the differences between different Lyapunov vectors and exponents become significant and the spreading of densities takes place in an increasing number of dimensions. This can be understood as the diffusion of the effect of the perturbation to the neighbouring degrees of freedom of the system. Thus we may conclude that: (i) the decay of fidelity for a local perturbation is independent of the size $N$ of the system for sufficiently large systems, but (ii) that it is faster than exponential since more and more degrees of freedom are beginning to contribute to the decay due to the diffusion of the perturbation effects (exponential decay is expected if only a single degree of freedom would be relevant). However, since the nature of the diffusion and the related properties of the Lyapunov vectors and the spectrum are very much system dependent, there is no universal decay of fidelity for the case of the local perturbation.

Our theoretical predictions will be demonstrated numerically in a generic chaotic classical many-body sys-

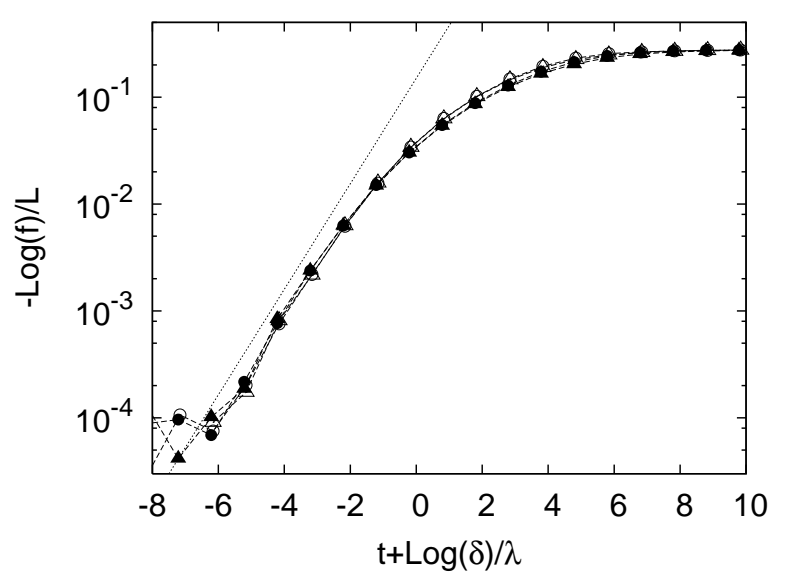

FIG. 3: Decay of fidelity as a function of time for the case of the continuous distribution, using the same represantation of scaled data as in Fig. 2] We show the data for $L=10$ (triangles) and 30 (circles) with perturbation strengths $\delta=$ $10^{-3}$ (empty symbols) and $10^{-4}$ (full symbols). The number of trajectories was $n_{t}=10^{7}$. We show the prediction (3) with arbitrarily chosen $\alpha$ and $\beta=2$ using the dotted line.

tem, namely a classical kicked Ising spin chain with the Hamiltonian (studied in the quantum version in [5])

$$
H=\sum_{i=1}^{L}\left[J_{i} s_{i}^{\mathrm{z}} s_{i+1}^{\mathrm{Z}}+\left(\sum_{j=-\infty}^{\infty} \delta(t-j)\right) K_{i} s_{i}^{\mathrm{x}}\right] .
$$

$L$ classical spin variables $\vec{s}_{i}$ take values on unit spheres, $\vec{s}_{i}^{2}=1$, and periodic boundary conditions are assumed $L+1 \equiv 1$. The coupling and kick parameters were normally set to constant values $J_{i}=2$ and $K_{i}=2$, respectively. The dimension of the phase space of such a system is $N=2 L$. The perturbation of the system was done by modifying the kicking strength $K_{i}^{\prime}=K_{i}+\delta V_{i}$. In the case of the global perturbation this was done for all $i$ uniformly, normally we set $V_{i}=1$, while in the case of the local perturbation, $V_{1}=1, V_{i \neq 1}=0$.

In order to demonstrate the differences between continuous and discontinuous distributions, we used two sets of initial densities. For the discontinuous case $\beta=1$, we used an initial uniform distribution of all spins on the upper hemispheres $s_{i}^{z}>0$. Even though the support for these distributions is not small in length as compared to a diameter of phase space, the fraction of the phase space volume that they cover, which gives the fidelity plateau $f(\infty)$, drops to 0 as $2^{-L}$ in the thermodynamic limit.

For the continuous density our choice of the distribution was a product of square normalized distributions $w\left(s^{z}\right)=\sqrt{3 / 8}\left(s^{z}+1\right)$ for all spins. This, however, does not imply that the distribution is a product distribution in the Lyapunov directions. Similarly to the case of discontinuous densities, fidelity plateau is again expected to drop to 0 in the large $L$ limit, namely $f(t \rightarrow \infty)=\left[2^{-1} \int_{-1}^{1} \mathrm{~d} s^{z} w\left(s^{z}\right)\right]^{L}=(3 / 8)^{L / 2}$. 


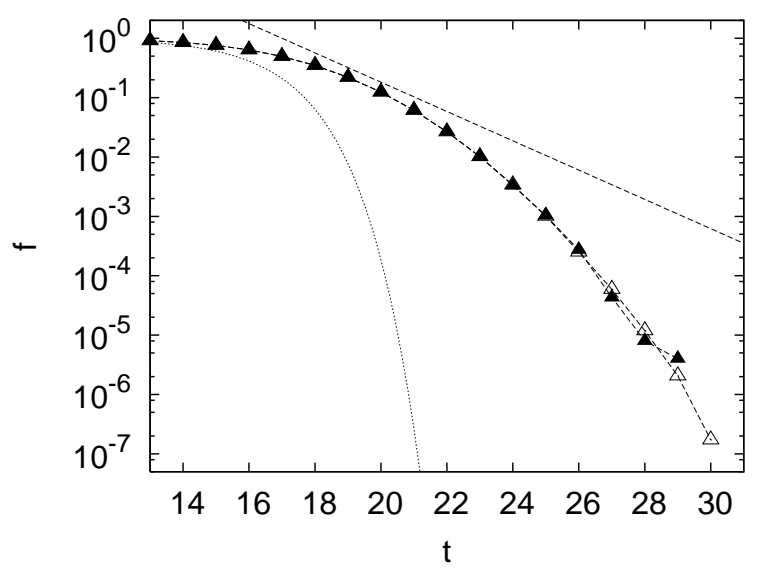

FIG. 4: Decay of fidelity as a function of time when a single spin is perturbed, and discontinuous initial density is chosen. We show the decay in the case of $L=100$ (empty, $n_{t} \approx 5.8 \times 10^{6}$ trajectories) and $L=1000$ (full triangles, $n_{t}=10^{6}$ ) with $\delta=10^{-4}$. The prediction (3) for extended perturbations with arbitrary $\alpha$ is shown as a dotted line and the (arbitrarily shifted) exponential decay with the maximum Lyapunov exponent of the system as a dashed line. As explained in the text, the actual decay falls in between the two types of behavior.

To be able to test the predictions we need to determine the Lyapunov spectrum of such a system. We show the positive part of the Lyapunov spectrum in Fig. 1 We checked that the negative part of the spectrum corresponds to the positive part and that the spectrum converges with time. We may see that the actual density of Lyapunov exponents divided by $L$ beautifully scales to the limiting density when $L$ is large enough. Such a behaviour is typically found for interacting many-particle systems, such as e.g. Fermi-Pasta-Ulam chain, Lorentz gas, etc. This justifies our assumption that the number of Lyapunov exponents in the neighbourhood of the largest Lyapunov exponent increases linearly with $N=2 L$, yet the maximum Lyapunov exponent converges with $N$.

We show the decay of fidelity for the discontinuous case and a global perturbation in Fig. [2] Using the appropriate scaling with respect to the number of spins $L=N / 2$ and perturbation strength $\delta$ as predicted in equation (3), where $\beta=1$, we may see that the doubly exponential behavior with the predicted dependence on $\delta$ and $N$ holds true. It should be noted that, while there is an observed plateau of fidelity when $N$ is still small, this plateau exponentially tends to 0 with $N$ and indeed the doubly exponential behavior of fidelity can be observed for all values of fidelity for large $N$.

Similar observations hold true for the continuous case where $\beta=2$ as shown in Fig. 3. For the same system, using continuous densities results in a faster form of decay with time and with quadratic dependence on the perturbation. In the continuous case we also observe expected numerical statistical fluctuations of fidelity at values close to 1 , especially for large $N$. This is because the initial density is represented by a finite number of trajectories and even when $t=0$ the estimation of fidelity will differ from 1. Nevertheless, the predicted scaling with $N$ and $\delta$ is observed along with the faster decay of fidelity with time as compared to discontinuous case.

For the case of a local perturbation with discontinuous densities as presented in Fig. 4 we observe a decay that lies in between the exponential behavior, as expected in systems with a single degree of freedom, and the doubly exponential behavior as is found in the global perturbation case. Details of such behavior are system dependent as they are determined by the diffusion of the effects of the perturbation to neighbouring degrees of freedom.

In addition we have tested our predictions for the disordered cases where the kicking strengths $K_{i}$ randomly varied from spin to spin, as well as the perturbations of the kicking strength $V_{i}$, however the results were again confirming our theory.

In conclusion, we have shown and explained that the decay of the classical Loschmidt echo in systems with a large number of degrees of freedom decays as a doubly exponential function for a global perturbation, and the decay rate depends on whether the phase space densities chosen are continuous or not. For the case of a local perturbation a system dependent form of decay is observed and justified that falls between exponential and doubly exponential behaviors. We acknowledge support by the grant P1-044 of Ministry of Science and Technology of Slovenia, and in part by the U.S. ARO grant DAAD1902-1-0086.
[1] J. Loschmidt, Sitzungsberichte der Akademie der Wissenschaften, Wien, 73, 128 (1876).

[2] A. Peres, Phys. Rev. A 30, 1610 (1984).

[3] H. M. Pastawski et al. Physica A 283, 166 (2000).

[4] R. A. Jalabert and H. M. Pastawski, Phys. Rev. Lett. 86, 2490 (2001).

[5] T. Prosen, Phys. Rev. E 65, 036208 (2002).
[6] T. Prosen and M. Žnidarič, J. Phys. A 35, 1455 (2002).

[7] B. Eckhardt, J. Phys. A: Math. Gen 36, 371 (2003).

[8] G. Benenti, G. Casati and G. Veble, Phys. Rev. E 67, 055202 (2003).

[9] G. Veble and T. Prosen, Phys. Rev. Lett. 92, 034101 (2004). 